\title{
LA CALIDAD DE VIDA DE LAS PERONAS MAYORES FRÁGILES INSTITUCIONALIZAS
}

\author{
Mirian Santamaría-Peláez \\ Universidad de Burgos \\ mspelaez@ubu.es \\ Jerónimo González-Bernal \\ Universidad de Burgos \\ jejavier@ubu.es \\ Josefa González-Santos \\ Universidad de Burgos \\ mjgonzalez@ubu.es \\ Raúl Soto-Cámara \\ Universidad de Burgos \\ rscamara@ubu.es
}

Recepción Artículo: 28 octubre 2020

Admisión Evaluación: 17 noviembre 2020

Informe Evaluador 1: 23 noviembre 2020

Informe Evaluador 2: 25 noviembre 2020

Aprobación Publicación: 27 noviembre 2020

\section{RESUMEN}

Estudio descriptivo y transversal que pretende estableces la relación de la situación de fragilidad en adultos mayores institucionalizados y la calidad de vida de los mismos en una muestra compuesta por 194 participantes.

La fragilidad se mide con la herramienta Short Physical Performance Battery y la calidad de vida con la escala GENCAT; ambas con buenas propiedades psicométricas.

Los resultados muestran que existen diferencias significativas entre los grupos de fragilidad en relación a la calidad de vida, tanto en su puntuación total como en cada una de sus subescalas. Además, tomando el valor numérico total de la escala SPPB, aparece una correlación estadísticamente significativa con la puntuación total en calidad de vida y con todas sus subescalas; de forma que, a medida que el estado de fragilidad avanza hacia la dependencia, la calidad de vida disminuye.

Los resultados sugieren que, el síndrome de fragilidad, además de tratamientos encaminados a paliar el deterioro físico y funcional de las personas mayores, debería ser abordado igualmente desde las esferas social y emocional.

Palabras clave: calidad de vida; fragilidad; síndrome de fragilidad; personas mayores; institucionalización 


\section{LA CALIDAD DE VIDA DE LAS PERONAS MAYORES FRÁGILES INSTITUCIONALIZAS}

\section{ABSTRACT}

Quality of life in frail institutionalized older people. Descriptive and transversal study that aims to establish the relationship of the situation of frailty in institutionalized older adults and their quality of life in a sample composed of 194 participants.

Frailty is measured with the Short Physical Performance Battery tool and quality of life with the GENCAT scale; both with good psychometric properties.

The results show that there are significant differences between frailty groups in relation to quality of life, both in their total score and in each of their subscales. In addition, taking the total numerical value of the SPPB scale, a statistically significant correlation appears with the total score in quality of life and with all its subscales; so that as the state of frailty moves toward dependence, quality of life decreases.

The results suggest that frailty syndrome, in addition to treatments aimed at alleviating the physical and functional deterioration of the elderly, should also be addressed from the social and emotional spheres.

Keywords: quality of life; frailty; frailty syndrome; elderly people; institutionalization

\section{INTRODUCCIÓN}

La presente investigación se centra en la calidad de vida, por una parte y, en la situación de fragilidad, por otra; en las personas mayores institucionalizadas.

De una parte, se entiende la calidad de vida como: "un estado de satisfacción general, derivado de la realización de las potencialidades de la persona. Posee aspectos subjetivos y aspectos objetivos. Es una sensación subjetiva de bienestar físico, psicológico y social. Incluye como aspectos subjetivos la intimidad, la expresión emocional, la seguridad percibida, la productividad personal y la salud objetiva. Como aspectos objetivos el bienestar material, las relaciones armónicas con el ambiente físico y social y con la comunidad, y la salud objetivamente percibida (Ardila, 2003)".

Por otra parte, la clasificación de tipologías de personas mayores en relación con la funcionalidad fue incluida en la Primera Conferencia de Prevención y Promoción de la Salud en la Práctica Clínica en España, y recogió Ios criterios que definen al anciano frágil como persona que conserva su independencia de forma inestable y que se halla en riesgo de pérdida funcional, bien por presentar una serie de factores de riesgo de deterioro, o bien porque ya manifiesta un deterioro funcional incipiente, aún reversible, que no causa una dependencia evidente (Abizanda y cols., 2011).

La fragilidad, por su parte, se considera en las diferentes definiciones como resultado de la disminución de la capacidad de reserva que conduce a situaciones de dependencia, al ingreso en una institución o a la muerte. Esta disminución tiene lugar en los niveles biomédicos y psicosociales (Redín, 1999).

Abizanda y Rodríguez (2014) contemplaron la siguiente definición de fragilidad: «síndrome biológico de disminución de la reserva funcional y resistencia a los estresores, debido al declive acumulado de múltiples sistemas fisiológicos que originan pérdida de la capacidad homeostática y vulnerabilidad a eventos adversos».

En los últimos años se ha comenzado a publicar estudios en residencias, como el estudio FINAL realizado en Cuenca y Albacete que arroja datos de cifras de fragilidad en residencias del $53,7 \%$ y el 68,8 \%, respectivamente (Abizanda y Rodríguez, 2014). Otros estudios fuera de España registran resultados similares con una prevalencia en residencias del 52,3 \% (Kojima, 2015).

La fragilidad se considera un factor de predicción de resultados adversos como: estado de salud, patologías asociadas, caídas, incapacidad, disminución de la calidad de vida, uso de los servicios sanitarios, institucionalización e incapacidad. Su prevalencia tiene un impacto importante en el área de la salud pública (Fried y cols., 2001; Fried, Ferrucci, Darer, Williamson y Anderson, 2004; Woo, Goggins, Sham y Ho, 2005; Abizanda y Rodríguez, 2014).

Existen trabajos que exploran las relaciones entre el estado cognitivo y emocional y la fragilidad física demostrando una asociación bidireccional entre ambos dominios. Se considera que los síntomas psicológicos 
empeoran el grado de fragilidad física y que la propia fragilidad física supone un riesgo para el empeoramiento del estado cognitivo y la depresión (Fielding, Sieber y Vellas, 2015). También en el plano mental y psicoafectivo se encontró la apatía, un serio síntoma neuropsiquiátrico asociado al deterioro cognitivo, al delirio y a la discapacidad, además de suponer por sí misma un factor predictor de mortalidad, en un estudio realizado con pacientes frágiles (Höltta y cols., 2012).

\section{OBJETIVOS DE LA INVESTIGACIÓN}

El objetivo de esta investigación radica en comprobar si existe relación entre la calidad de vida y la situación de fragilidad en las personas mayores institucionalizadas.

\section{MÉTODO}

Estudio multicéntrico en el que participaron 194 personas institucionalizadas en 4 residencias para mayores; para lo que se realizó una única toma de datos en los propios centros de trabajo. Así se trata de un estudio transversal, no experimental y descriptivo, que pretende establecer las relaciones entre las variables, que no conlleva intervención con los participantes y tampoco pretende establecer relaciones de causalidad entre las variables.

Los datos fueron anonimizados, de manera que se preserva en todo momento la confidencialidad de los mismos y se garantiza la imposibilidad de identificar a ningún participante. Este procedimiento fue aprobado por el Comité de Ética de la Universidad de Burgos (IR 11/2018).

Las variables que conforman este estudio son la calidad de vida, medida con la escala GENCAT y el estado de fragilidad, medido con LA ESCALA Short Physical Performance Battery (SPPB)

El Instituto Catalán de Asistencia y Servicios Sociales (ICASS), en colaboración con el Instituto de Integración de la Comunidad (INICO) de la Universidad de Salamanca desarrollaron y validaron la escala de valoración de la calidad de vida GENCAT y tiene la finalidad de identificar el perfil de calidad de vida de la persona para poder desarrollar planes individuales de apoyo. Se administra de forma individual a adultos a partir de 18 años. Los informadores deben ser profesionales de los servicios sociales que conozcan a la persona al menos desde hace tres meses. Requiere un tiempo entre 10 y 15 minutos para su cumplimentación.

Propone una evaluación objetiva de la calidad de vida en base a ocho dimensiones (bienestar emocional, bienestar físico, bienestar material, relaciones interpersonales, inclusión social, desarrollo personal, autodeterminación y derechos). Cada dimensión contiene una serie de ítems, los cuales se valoran entre 1 y 4.

La validación que concluyó que «la escala GENCAT es un instrumento válido y fiable que sirve a los objetivos planteados: la medición objetiva de la calidad de vida de usuarios de servicios sociales» (Departamento de Acción Social y Ciudadanía de la Generalitat de Cataluña, 2009), se llevó a cabo con una muestra de 3029 usuarios de servicios sociales de la Comunidad Autónoma de Cataluña. El grupo más representativo fue el formado por las personas mayores que se encontraban en residencias (44,70\%). El coeficiente de Cronbach de la escala con todas sus subescalas es de 0,916; por lo tanto, tiene una adecuada consistencia interna (Verdugo, Arias, Gómez y Schalock, 2009).

La escala SPPB, es una prueba de ejecución muy utilizada en el medio geriátrico que resulta bastante sencilla y rápida en su aplicación y no requiere ningún tipo de equipamiento. Es el instrumento que el Documento consenso sobre fragilidad y caídas en personas mayores propone como primera opción para valorar la existencia 0 no de fragilidad (Ministerio de Sanidad, Servicios Sociales e Igualdad, 2014).

Se trata de una herramienta validada ampliamente en diferentes poblaciones y ajustada por edad, sexo y comorbilidad; así, supone un instrumento muy fiable para detectar fragilidad y predecir la discapacidad (Cabrero, Cabañero y Ramos, 2012; Poveda, 2014; Poveda, 2015; Pavasini y cols, 2016; Gómez, Curcio, Alvarado, Zunzunegui y Guralnik, 2013). 


\section{LA CALIDAD DE VIDA DE LAS PERONAS MAYORES FRÁGILES INSTITUCIONALIZAS}

Consta de tres pruebas diferenciadas:

- Equilibrio: con los pies juntos, en semitándem y en tándem; puntúa entre 0 y 4.

- Velocidad de la marcha: sobre 2,4 04 metros; puntúa entre 0 y 4.

- Levantarse y sentarse en una silla cinco veces; puntúa entre 0 y 4.

La puntuación total del SPPB estará entre 0 y 12, siendo 0 la peor situación y la puntuación por debajo de 10 indicativa de fragilidad y de un elevado riesgo de caídas (Ministerio de Sanidad, Servicios Sociales e Igualdad, 2014).

El Programa ViviFrail de entrenamiento físico multicomponente para la prevención de la fragilidad y las caídas en mayores de 70 años, cofinanciado por la Unión Europea y perteneciente a Erasmus+, propone además los siguientes puntos de corte para el test SPPB (Proyecto ViviFrail):

- Limitación severa: DEPENDIENTE 0 DISCAPACITADO: SPPB 0-3.

- Limitación moderada: FRÁGIL: SPPB 4-6.

- Limitación leve: PREFRÁGIL: SPPB 7-9.

- Limitación mínima: AUTÓNOMO 0 ROBUSTO: SPPB 10-12.

En el presente estudio, se utilizan los datos del SPPB de ambas formas, por lo que se trataría de una variable cuantitativa si se tiene en cuenta la puntuación numérica y de una variable cualitativa polinómica si se tienen en cuenta los 4 grupos en los que se divide a los participantes en función del grado de fragilidad o dependencia.

\section{RESULTADOS}

Las pruebas que se realizan son no paramétricas ya que la prueba de Kolmogorof-Smirnov es significativa ( $\mathrm{p} \leq$.05). Par la relación cuantitativa-cuantitativa se utiliza correlación de Spearman y para la relación cuantitativa-cualitativa la Anova, según las consideraciones de Blanca, Alarcón, Arnau, Bono y Bendayan (2017).

La ANOVA muestra que existen diferencias significativas entre los grupos de fragilidad establecidos en el SPPB en relación a las puntuaciones de calidad de vida total y de cada una de sus dimensiones $(, 016>p>, 001)$.

Para profundizar en las diferencias que existen entre los grupos se realiza un análisis post hoc que ofrece los siguientes resultados (tabla 1):

- El grupo de personas dependientes tiene diferencias significativas con el resto de los grupos en todas las dimensiones de calidad de vida y en la puntuación total; excepto con el grupo prefrágil en bienestar material y bienestar físico, y con los grupos frágil y robusto en derechos.

- Las personas frágiles, además de las relaciones con el grupo dependiente ya descritas, muestran diferencias significativas con el grupo de las personas robustas en relaciones interpersonales, bienestar físico, inclusión social y calidad de vida total.

- Las personas prefrágiles, además de lo anterior, presentan diferencias significativas con el grupo robusto en lo referente al bienestar físico. 
Tabla 1. Pruebas post hoc. Comparaciones múltiples. Bonferroni. Fragilidad-bienestar emocional

\begin{tabular}{|c|c|c|c|c|c|c|c|}
\hline \multirow{2}{*}{$\begin{array}{l}\text { Variabl } \\
\text { e } \\
\text { depend. }\end{array}$} & \multirow{2}{*}{$\begin{array}{l}\text { (I) } \\
\text { Rango } \\
\text { s SPPB }\end{array}$} & \multirow{2}{*}{$\begin{array}{l}(\mathbf{J}) \\
\text { Rangos } \\
\text { SPPB }\end{array}$} & \multirow{2}{*}{$\begin{array}{l}\text { Diferenc } \\
\text { ia de } \\
\text { medias } \\
\text { (I-J) }\end{array}$} & \multirow{2}{*}{$\begin{array}{l}\text { Error } \\
\text { están } \\
\text { dar }\end{array}$} & \multirow[t]{2}{*}{$\begin{array}{l}\text { Sig } \\
\text {. }\end{array}$} & \multicolumn{2}{|c|}{$\begin{array}{c}\text { Intervalo de } \\
\text { confianza al } 95 \% \\
\end{array}$} \\
\hline & & & & & & Lím inf. & Lím sup. \\
\hline \multirow{8}{*}{$\begin{array}{l}\text { GENCA } \\
\mathrm{T} \\
\text { bienestar } \\
\text { emocion } \\
\text { al }\end{array}$} & \multirow{4}{*}{$\begin{array}{l}\text { Depen } \\
\text { d. }\end{array}$} & Frágil & $-5,360^{*}$ & ,994 & ,00 & $-8,01$ & $-2,71$ \\
\hline & & & & & 0 & & \\
\hline & & Prefrágil & $-4,248^{*}$ & 1,155 & $\begin{array}{l}, 00 \\
2\end{array}$ & $-7,33$ & $-1,17$ \\
\hline & & Robusto & $-8,177^{*}$ & 1,466 & $\begin{array}{l}, 00 \\
0\end{array}$ & $-12,09$ & $-4,27$ \\
\hline & Frágil & $\begin{array}{l}\text { Dependi } \\
\text { ente }\end{array}$ & $5,360^{*}$ & ,994 & $\begin{array}{l}00 \\
0\end{array}$ & 2,71 & 8,01 \\
\hline & $\begin{array}{l}\text { Prefrág } \\
\text { il }\end{array}$ & $\begin{array}{l}\text { Dependi } \\
\text { ente }\end{array}$ & $4,248^{*}$ & 1,155 & $\begin{array}{l}00 \\
2\end{array}$ & 1,17 & 7,33 \\
\hline & \multirow[t]{2}{*}{$\begin{array}{l}\text { Robust } \\
\text { o }\end{array}$} & $\begin{array}{l}\text { Dependi } \\
\text { ente }\end{array}$ & $8,177^{*}$ & 1,466 & $\begin{array}{l}, 00 \\
0\end{array}$ & 4,27 & 12,09 \\
\hline & & & & & & $\begin{array}{l}\text { Lím } \\
\text { inf. }\end{array}$ & Lím sup. \\
\hline \multirow{9}{*}{$\begin{array}{l}\text { GENCA } \\
\mathrm{T} \\
\text { relacion } \\
\text { es } \\
\text { interper. }\end{array}$} & \multirow{3}{*}{$\begin{array}{l}\text { Depen } \\
\text { d. }\end{array}$} & Frágil & $-4,042^{*}$ & 1,033 & ,001 & $-6,80$ & $-1,29$ \\
\hline & & Prefrágil & $-5,907^{*}$ & 1,200 &, 000 & $-9,11$ & $-2,71$ \\
\hline & & Robusto & $-8,346^{*}$ & 1,523 &, 000 & $-12,41$ & $-4,29$ \\
\hline & \multirow[t]{2}{*}{ Frágil } & $\begin{array}{l}\text { Dependie } \\
\text { nte }\end{array}$ & $4,042^{*}$ & 1,033 & ,001 & 1,29 & 6,80 \\
\hline & & Robusto & $-4,304$ & 1,619 &, 051 & $-8,62$ &, 01 \\
\hline & $\begin{array}{l}\text { Prefrág } \\
\text { il }\end{array}$ & $\begin{array}{l}\text { Dependie } \\
\text { nte }\end{array}$ & $5,907^{*}$ & 1,200 &, 000 & 2,71 & 9,11 \\
\hline & \multirow[t]{3}{*}{$\begin{array}{l}\text { Robust } \\
\text { o }\end{array}$} & $\begin{array}{l}\text { Dependie } \\
\text { nte }\end{array}$ & $8,346^{*}$ & 1,523 &, 000 & 4,29 & 12,41 \\
\hline & & Frágil & 4,304 & 1,619 &, 051 &,- 01 & 8,62 \\
\hline & & & & & & $\begin{array}{l}\text { Lím } \\
\text { inf. }\end{array}$ & Lím sup. \\
\hline \multirow{5}{*}{$\begin{array}{l}\text { GENCA } \\
\mathrm{T} \\
\text { bienestar } \\
\text { material }\end{array}$} & \multirow{2}{*}{$\begin{array}{l}\text { Depen } \\
\text { d. }\end{array}$} & Frágil & $-1,242^{*}$ & ,416 & ,019 & $-2,35$ &,- 13 \\
\hline & & Robusto & $-1,739^{*}$ & ,614 &, 031 & $-3,38$ &,- 10 \\
\hline & Frágil & $\begin{array}{l}\text { Dependie } \\
\text { nte }\end{array}$ & $1,242^{*}$ & ,416 & ,019 &, 13 & 2,35 \\
\hline & \multirow[t]{2}{*}{$\begin{array}{l}\text { Robust } \\
\text { o }\end{array}$} & $\begin{array}{l}\text { Dependie } \\
\text { nte }\end{array}$ & $1,739^{*}$ & ,614 &, 031 &, 10 & 3,38 \\
\hline & & & & & & $\begin{array}{l}\text { Lím } \\
\text { inf. }\end{array}$ & Lím sup. \\
\hline
\end{tabular}




\section{LA CALIDAD DE VIDA DE LAS PERONAS MAYORES FRÁGILES INSTITUCIONALIZAS}

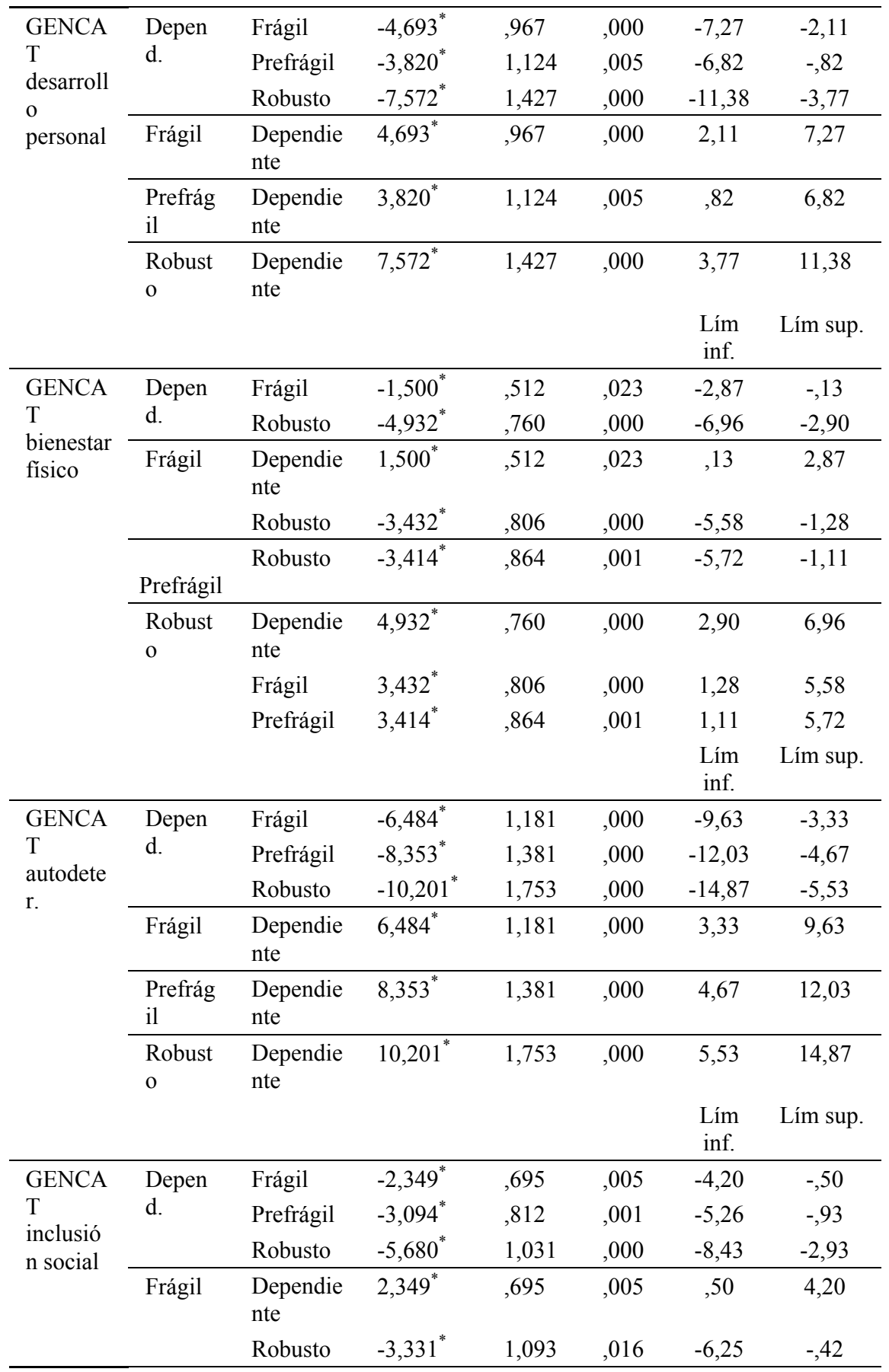




\begin{tabular}{|c|c|c|c|c|c|c|c|}
\hline & $\begin{array}{l}\text { Prefrág } \\
\text { il }\end{array}$ & $\begin{array}{l}\text { Dependie } \\
\text { nte }\end{array}$ & $3,094^{*}$ & 812 & ,001 & ,93 & 5,26 \\
\hline & $\begin{array}{l}\text { Robust } \\
\text { o }\end{array}$ & $\begin{array}{l}\text { Dependie } \\
\text { nte }\end{array}$ & $5,680^{*}$ & 1,031 &, 000 & 2,93 & 8,43 \\
\hline & & Frágil & $3,331^{*}$ & 1,093 &, 016 & ,42 & 6,25 \\
\hline & & & & & & $\begin{array}{l}\text { Lím } \\
\text { inf. }\end{array}$ & Lím sup. \\
\hline \multirow{3}{*}{$\begin{array}{l}\text { GENCAT } \\
\text { derechos }\end{array}$} & Depend. & Prefrágil & $-2,285^{*}$ & 845 &, 045 & $-4,54$ &,- 03 \\
\hline & $\begin{array}{l}\text { Prefrág } \\
\text { il }\end{array}$ & $\begin{array}{l}\text { Dependie } \\
\text { nte }\end{array}$ & $2,285^{*}$ &, 845 &, 045 &, 03 & 4,54 \\
\hline & & & & & & Lím inf. & $\begin{array}{l}\text { Lím } \\
\text { sup. }\end{array}$ \\
\hline \multirow[t]{9}{*}{$\begin{array}{l}\text { GENCA } \\
\mathrm{T} \text { total }\end{array}$} & Depend & Frágil & $\begin{array}{l}- \\
24.65659\end{array}$ & 4,86719 & $\begin{array}{l}, 00 \\
0\end{array}$ & $\begin{array}{c}- \\
376333\end{array}$ & $\begin{array}{c}- \\
116799\end{array}$ \\
\hline & & $\begin{array}{l}\text { Prefrá } \\
\text { gil }\end{array}$ & $\begin{array}{l}- \\
30,31568\end{array}$ & 5,68939 & $\begin{array}{l}, 00 \\
0\end{array}$ & $\begin{array}{c}- \\
45,4845\end{array}$ & $\begin{array}{c}- \\
15,1468\end{array}$ \\
\hline & & $\begin{array}{l}\text { Robust } \\
\text { o }\end{array}$ & $\begin{array}{l}- \\
49,07326\end{array}$ & 7,22252 & $\begin{array}{l}, 00 \\
0\end{array}$ & $\begin{array}{c}- \\
68,3297\end{array}$ & $\begin{array}{c}- \\
29,8168\end{array}$ \\
\hline & Frágil & $\begin{array}{l}\text { Depen } \\
\text { d. }\end{array}$ & 24,65659 & 4,86719 & $\begin{array}{l}, 00 \\
0\end{array}$ & 11,6799 & 37,6333 \\
\hline & & $\begin{array}{l}\text { Robust } \\
\mathrm{o}\end{array}$ & $\begin{array}{l}- \\
24,41667\end{array}$ & 7,65673 & $\begin{array}{l}, 01 \\
0\end{array}$ & $\begin{array}{c}- \\
44,8308\end{array}$ & $-4,0026$ \\
\hline & $\begin{array}{l}\text { Prefrág } \\
\text { il }\end{array}$ & $\begin{array}{l}\text { Depen } \\
\text { d. }\end{array}$ & 30,31568 & 5,68939 & $\begin{array}{l}, 00 \\
0\end{array}$ & 15,1468 & 45,4845 \\
\hline & $\begin{array}{l}\text { Robust } \\
\text { o }\end{array}$ & $\begin{array}{l}\text { Depen } \\
\text { d. }\end{array}$ & ${ }_{*}^{49,07326}$ & 7,22252 & $\begin{array}{l}, 00 \\
0\end{array}$ & 29,8168 & 68,3297 \\
\hline & & Frágil & 24,41667 & 7,65673 & $\begin{array}{l}, 01 \\
0\end{array}$ & 4,0026 & 44,8308 \\
\hline & & & & & & Lím inf. & $\begin{array}{l}\text { Lím } \\
\text { sup. }\end{array}$ \\
\hline
\end{tabular}

* La diferencia de medias es significativa en el nivel 0,05

Comparando las puntuaciones de la escala GENCAT y las puntuaciones de la escala SPPB, totales y de cada subescala en ambos casos, se aprecia que en todos los casos aparecen correlaciones significativas entre ellas al nivel (,004 > p > ,001), con los coeficientes de correlación que en cada uno de ellos se indica en la tabla 2.

Se trata de una correlación positiva en todos los casos, lo que indica que, a mayor puntuación en calidad de vida, mayor puntuación en fragilidad; esto es, cuanto mayor es la calidad de vida percibida, más se acerca la persona al estado de robustez en el SPPB. 


\section{LA CALIDAD DE VIDA DE LAS PERONAS MAYORES FRÁGILES INSTITUCIONALIZAS}

Tabla 2. Rho de Spearman. Fragilidad-calidad de vida.

\begin{tabular}{|c|c|c|c|c|c|}
\hline & & $\begin{array}{l}\text { SPPB } \\
\text { equil. }\end{array}$ & $\begin{array}{l}\text { SPPB } \\
\text { veloc. }\end{array}$ & $\begin{array}{c}\text { SPPB } \\
\text { levant } \\
\text {. }\end{array}$ & $\begin{array}{c}\text { SPPB } \\
\text { total }\end{array}$ \\
\hline \multirow{3}{*}{$\begin{array}{l}\text { Bienestar } \\
\text { emocional }\end{array}$} & Coeficiente de correlación &, $421^{* *}$ &, $414^{* *}$ &, $388^{* *}$ &, $440^{* *}$ \\
\hline & Sig. (bilateral) & 000 &, 000 &, 000 &, 000 \\
\hline & $\mathrm{N}$ & 194 & 194 & 194 & 194 \\
\hline \multirow{3}{*}{$\begin{array}{l}\text { Relaciones } \\
\text { interpersonales }\end{array}$} & Coeficiente de correlación & $477^{* *}$ &, $414^{* *}$ &, $432^{* *}$ &, $478^{* *}$ \\
\hline & Sig. (bilateral) &, 000 &, 000 &, 000 & ,000 \\
\hline & $\mathrm{N}$ & 194 & 194 & 194 & 194 \\
\hline \multirow{3}{*}{$\begin{array}{l}\text { Bienestar } \\
\text { material }\end{array}$} & Coeficiente de correlación &, $370^{* *}$ & $238^{* *}$ & $207^{* *}$ &, $315^{* *}$ \\
\hline & Sig. (bilateral) & ,000 &, 001 & ,004 &, 000 \\
\hline & $\mathrm{N}$ & 194 & 194 & 194 & 194 \\
\hline \multirow{3}{*}{$\begin{array}{l}\text { Desarrollo } \\
\text { personal }\end{array}$} & Coeficiente de correlación & $466^{* *}$ &, $332^{* *}$ &, $372^{* *}$ &, $420^{* *}$ \\
\hline & Sig. (bilateral) &, 000 &, 000 &, 000 & ,000 \\
\hline & $\mathrm{N}$ & 194 & 194 & 194 & 194 \\
\hline \multirow{3}{*}{$\begin{array}{l}\text { Bienestar } \\
\text { físico }\end{array}$} & Coeficiente de correlación &, $402^{* *}$ &, $367^{* *}$ &, $414^{* *}$ &, $410^{* *}$ \\
\hline & Sig. (bilateral) &, 000 &, 000 &, 000 &, 000 \\
\hline & $\mathrm{N}$ & 194 & 194 & 194 & 194 \\
\hline \multirow{3}{*}{$\begin{array}{l}\text { Autodetermina } \\
\text { ción }\end{array}$} & Coeficiente de correlación &, $556^{* *}$ & $446^{* *}$ & $460^{* *}$ &, $540^{* *}$ \\
\hline & Sig. (bilateral) & ,000 &, 000 &, 000 &, 000 \\
\hline & $\mathrm{N}$ & 194 & 194 & 194 & 194 \\
\hline \multirow{3}{*}{$\begin{array}{l}\text { Inclusión } \\
\text { social }\end{array}$} & Coeficiente de correlación & $450^{* *}$ &, $367^{* *}$ &, $390^{* *}$ &, $436^{* *}$ \\
\hline & Sig. (bilateral) &, 000 &, 000 &, 000 &, 000 \\
\hline & $\mathrm{N}$ & 194 & 194 & 194 & 194 \\
\hline \multirow[t]{3}{*}{ Derechos } & Coeficiente de correlación &, $457^{* *}$ & $295^{* *}$ &, $351^{* *}$ &, $403^{* *}$ \\
\hline & Sig. (bilateral) &, 000 &, 000 &, 000 &, 000 \\
\hline & $\mathrm{N}$ & 194 & 194 & 194 & 194 \\
\hline \multirow[t]{3}{*}{ GENCAT total } & Coeficiente de correlación & $\begin{array}{c}, 580^{*} \\
*\end{array}$ & $\begin{array}{c}, 466^{*} \\
*\end{array}$ & $\begin{array}{c}, 510^{*} \\
*\end{array}$ & $\begin{array}{c}561^{*} \\
*\end{array}$ \\
\hline & Sig. (bilateral) &, 000 &, 000 &, 000 &, 000 \\
\hline & $\mathrm{N}$ & 194 & 194 & 194 & 194 \\
\hline
\end{tabular}

** La correlación es significativa en el nivel 0,01 (bilateral) 


\section{DISCUSIÓN}

Este estudio obtiene unos datos que comprueban la correlación entre la fragilidad y la calidad de vida, de manera que a medida que la fragilidad disminuye, la calidad de vida aumenta, lo que se refleja en la puntuación total de la escala GENCAT y en la puntuación de cada una de sus dimensiones.

Estos resultados apuntan en la misma dirección que otros estudios previos que también mostraban esta relación, como el de Chamberlain y cols. (2016) que afirman que la fragilidad influye en la calidad de vida (Mello, Engstrom y Alves, 2014), y el estudio FRADEA que presenta la mala calidad de vida como una característica de la fragilidad, lo que fue confirmado en el estudio de Toledo (Abizanda y Rodríguez, 2014). Paralelamente, la sarcopenia incluye en la definición propuesta por el Grupo Europeo de Trabajo sobre la Sarcopenia en Personas de Edad Avanzada (EWGSOP) una calidad de vida deficiente (Jurado, 2015).

El tornado de la fragilidad (Centro Nacional de Excelencia Tecnológica y Salud (CENETEC), 2014) presenta la pobreza y el abandono social como fuerzas que envuelven al anciano frágil conduciéndolo a la discapacidad funcional y la muerte.

Aspectos como las relaciones interpersonales o familiares también han sido estudiados obteniendo resultados en la misma línea (Sousa y Dos Santos, 2014). El escaso apoyo social se presenta también como un factor de riesgo para el deterioro funcional y la fragilidad (Cánovas, Martín, Solsona y Chávez, 2011). Todos estos aspectos se encuentran, de una u otra manera, presentes en las dimensiones de calidad de vida que se han valorado.

La muestra que ha sido utilizada en este estudio presenta ciertas limitaciones ya que está caracterizada por ser no probabilística y de conveniencia, lo cual no posibilita que los resultados puedan ser generalizables al resto de la población. Sin embargo, los resultados obtenidos que alcanzan los objetivos previstos en un inicio sugieren que sería conveniente ampliar dicha muestra para que de esa forma la investigación sí pudiera ser representativa.

\section{CONCLUSIONES}

Los datos descritos en los resultados del estudio muestran que existen diferencias entre todos los grupos en varias de las dimensiones de calidad de vida y en la puntuación total. Establecer estas diferencias implica, como ya se ha mencionado, poder detectar a las personas que pertenecen a un grupo antes de que pasen al siguiente y continúen avanzando hacia la dependencia.

El objetivo relacionado con este apartado acerca de comprobar si existe relación entre la fragilidad y; la ansiedad, la depresión y la apatía se considera cumplido. Se ha podido relacionar todas ellas con la fragilidad de forma que las tres muestran una correlación significativa; así, a medida que la fragilidad aumenta y avanza hacia el extremo de la dependencia, la ansiedad, la depresión y la apatía también aumentan.

Esta relación de la fragilidad con el estado social y emocional de la persona hace pensar que además de los tradicionales abordajes sobre la funcionalidad que se centran en la parte más física, sería conveniente proponer intervenciones que incidan también sobre esta situación socioemocional negativa que a buen seguro afecta al estado general de salud y bienestar de las personas.

\section{REFERENCIAS BIBLIOGRÁFICAS}

Abizanda Soler, P., Sánchez Jurado, P., Romero, L., Paterna, G., Martínez Sánchez, E. y Atienzar Núñez, P. (2011). Prevalence of frailty in a Spanish elderly population: the frailty and dependence in Albacete Study. Journal of the American Geriatrics Society, 59(7), 1356-1359.

Abizanda Soler, P. y Rodríguez Mañas, L. (2014). Evolución histórica del término 'fragilidad'. Realidad actual. En P. Ramos Cordero (coord.), Guía de buena práctica clínica en geratría. Fragilidad y nutrición en el anciano (págs. 15-30). Madrid, España: International Marketing and Communication.

Ardila, R. (2003). Calidad de vida: una definición integradora. Revista Latinoamericana de psicología, 35(2), 161-164. 


\section{LA CALIDAD DE VIDA DE LAS PERONAS MAYORES FRÁGILES INSTITUCIONALIZAS}

Blanca, M. J., Alarcón, R., Arnau, J., Bono, R. y Bendayan, R. (2017). Non-normal data: Is ANOVA still a valid option? Psicothema, 29(4), 552-557.

Cabrero García, J., Cabañero Martínez, M. J., Ramos Pichardo, J. D. y Reig Ferrer, A. (2012). Valores de referencia de la Short Physical Performance Battery para pacientes de 70 y más años en atención primaria de salud. Atención Primaria, 44(9), 540-548.

Cánovas, C., Martín, A., Solsona, S. y Chávez, F. (2011). Prevención de la fragilidad: anciano frágil y actividad física. Infogeriatría, 2011(1), 19-24.

Medina, J. H. (coord.). (2014). Diagnóstico y tratamiento del síndrome de fragilidad en el adulto mayor. Guía de referencia rápida. Catálogo maestro de guía de práctica clínica. Centro Nacional de Excelencia Tecnológica y Salud (CENETEC). México D.F.

Chamberlain, A. M., Sauver, J. L., Jacobson, D. J., Manemann, S. M., Fan, C., Roger, V. L., ... Finney, L. J. (2016). Social and behavioural factors associated with frailty trajectories in a population-based cohort of older adults. BMJ Open, 6(5), e011410. Doi: 10.1136/bmjopen-2016-011410.

Fielding, R. A., Sieber, C. y Vellas, B. (eds.) (2015). Frailty: Pathophysiology, Phenotype and Patient Care. 83rd Nestlé Nutrition Institute Workshop celebrado en Barcelona en marzo de 2014. Basilea, Suiza: Karger (Clinical Nutrition Series, 83).

Fried, L., Ferrucci, L., Darer, J., Williamson, J. y Anderson, G. (2004). Untangling the concepts of disability, frailty and comorbidity: implications for improved targeting and care. The Journals of Gerontology. Series A: Biological Sciences and Medical Sciences, 59(3), M255-M263.

Fried, L. P., Tangen, C. M., Walston, J. D., Newman, A. B., Hirch, A., Gottdiener, J., ... McBurnie, M. A. (2001). Frailty in older adults: evidence for a phenotype. Journals of Gerontology. Series A: Biological Sciences and Medical Sciences, 56A(3), M146-M156.

Gómez, J. F., Curcio, C. L., Alvarado, B., Zunzunegui, M. V. y Guralnik, J. (2013). Validity and reliability of the Short Physical Performance Battery (SPPB): a pilot study on mobility in the Colombian Andes. Colombia Médica, 44(3), 165-171.

Höltta, E. H., Laakkonen, M.-L., Laurila, J. V., Strandberg, T. E., Tilvis, R. S. y Pitkälä, K. H. (2012). Apathy: prevalence, associated factors, and prognostic value among frail, older inpatients. Journal of the American Medical Directors Association, 13(6), 541-545.

Jurado Ledesma, I. (2015). Sarcopenia y desempeño ocupacional de las actividades básicas de la vida diaria en mayores institucionalizados. Revista Asturiana de Terapia Ocupacional, 2015(12), $22-29$.

Kojima, G. (2015). Prevalence of Frailty in Nursing Homes: A Systematic Review and Meta-Analysis. Journal of the American Medical Directors Association, 16(11), 940-945.

Mello, C., Engstrom, E. y Alves, L. (2014). Health-related and socio-demographic factors associated with frailty in the elderly: a systematic literature review. Cadernos de Saúde Pública, 30(6), 1143-1168.

Ministerio de Sanidad, Servicios Sociales e Igualdad. (2014). Documento consenso sobre fragilidad y caídas. Estrategia de promoción de la Salud y Prevención en el SNS. Madrid, España: Ministerio de Sanidad, Servicios Sociales e Igualdad Centro de Publicaciones.

Pavasini, R., Guralnik, J., Brown, J. C., di Bari, M., Cesari, M., Landi, F., ... Campo, G. (2016). Short Physical Performance Battery and all-cause mortality: systematic review and meta-analysis. BMC Medicine, 14(1), 215.

Poveda Asensio, V. (2014/2015). Recopilación de test de campo para la valoración de la condición física en mayores (trabajo final de grado). Universidad Miguel Hernández. Recuperado de: https://pdfs.semanticscholar.org/170c/416cce7a2dbb4b76164e7b2aafa76f1dfeb6.pdf

Proyecto ViviFrail. (s.f.). www.vivifrail.com. Recuperado de: http://www.vivifrail.com/es

Redín Larraz, J. M. (1999). Valoración geriátrica integral (I). Avaluación del paciente geriátrico y concepto de fragilidad. ANALES Sistema Sanitario Navarra, 22(Supl 1), 41-50. 
Sousa, M. y Dos Santos, M. (2014). Factores asociados al síndrome de fragilidad en ancianos residentes en área urbana. Revista Lationamericana Erfermagem, 22(5), 874-882.

Verdugo Alonso, M. Á., Arias Martínez, B., Gómez Sánchez, L. E. y Schalock, R. L. (2009). Escala GENCAT: Manual de aplicación de la Escala GENCAT de Calidad de vida. Barcelona, España: Generalitat de Catalunya.

Woo, J., Goggins, W., Sham, A. y Ho, S. C. (2005). Social determinants of frailty. Gerontology, 51(6), 402-408. 
